\title{
Hastes maiores de pupunheira produzidas no Vale do Ribeira são mais produtivas
}

\author{
Pejibaye's larger stems produced in the Vale do Ribeira are more productives
}

\author{
Gabriel Henrique dos Santos Silva Salgado ${ }^{*}$, Samuel Ferrari ${ }^{2}$, Dariane Beatriz Schoffen Enke ${ }^{3}$ \\ ${ }^{1}$ Universidade Estadual Paulista, Faculdade de Ciências Agrárias e Veterinárias, Jaboticabal, SP, Brasil. *Autor para correspondência: \\ gabriel_salgado01@hotmail.com \\ ${ }^{2}$ Universidade Estadual Paulista, Faculdade de Ciências Agrárias e Tecnológicas, Dracena, SP, Brasil. \\ ${ }^{3}$ Universidade Estadual Paulista, Registro, SP, Brasil.
}

Submissão: 04/06/2019 / Aceite: 22/06/2020

\begin{abstract}
RESUMO
O interesse pela cultura da pupunha começou a crescer após a década de 70 , tendo em vista as qualidades agronômicas, industriais e comerciais. O Estado de São Paulo possui destaque como um dos maiores produtores de pupunha do país, sendo a maior concentração no Vale do Ribeira. Objetivou-se com este trabalho avaliar características de desenvolvimento vegetativo das plantas e produtividade de palmito em função do momento de corte das hastes em cultivo com diferentes densidades populacionais, para o segundo ano de corte. O delineamento experimental utilizado foi o de blocos casualizados em esquema fatorial $2 \times 5$ e quatro repetições, sendo os tratamentos com cinco classes de altura de corte do estipe $(1,60 \mathrm{~m}, 1,90 \mathrm{~m}, 2,20 \mathrm{~m}, 2,50 \mathrm{~m}$ e $2,80 \mathrm{~m})$ e duas densidades de plantas $(5.000$ e 10.000 plantas $\mathrm{ha}^{-1}$ ). O experimento foi conduzido na fazenda do Campus Experimental de Registro da UNESP. As análises foram realizadas em dois momentos, março e setembro de 2016 . Hastes de $2,8 \mathrm{~m}$ colhidas no mês de março obtiveram maior diâmetro do caule, número de folhas e quantidade total de palmito produzido. Hastes com a mesma altura de corte, porém colhidas no mês de setembro proporcionaram maior produção de palmito picado, rodela, tolete e quantidade total de palmito pupunha.
\end{abstract}

PALAVRAS-CHAVE: Bactris gasipaes, densidade populacional, palmito, produtividade.

\begin{abstract}
The interest for pejibaye crops started to grow after the 70's decade, due to the agronomic qualities, ecological, industrials and commercials. The São Paulo State is highlighted as one of the biggest pejibaye growers of the country, most concentrated at Vale do Ribeira. It was objectified with this work to evaluate characteristics of vegetative growth of plants as well as the ideal cutting time for stems in cultivation with different population densities, for the second year of cutting, aiming at the heart-of-palm yield of pejibaye. The experimental design was used in a $2 \times 5$ factorial, with treatments with five classes of cutting height $(1,60 \mathrm{~m}, 1,90 \mathrm{~m}, 2,20 \mathrm{~m}, 2,50 \mathrm{~m}$ and $2,80 \mathrm{~m})$ and two densities of plants $\left(5,000\right.$ and 10,000 plants ha $\left.{ }^{-1}\right)$. The experiment was led on Campus Experimental de Registro UNESP's farm. The analyzes were performed on march 16th and 17th of 2016 and September 01st e 19th of the same year. Stems of pejibaye at cutting height of $2,8 \mathrm{~m}$ and harvested in march obtained a larger number of leaves, a larger diameter of the stem in relation to the other heights and larger total amount of heart-of-palm produced. Stems with the same cutting height, but harvested in the month of September, provided greater production of minced hearts-of-palm, slice, thole and total amount of pejibaye heart-of-palm.
\end{abstract}

KEYWORDS: Bactris gasipaes, population density, heart-of-palm, yield.

\section{INTRODUÇÃO}

Bactris gasipaes Kunth, também conhecida como pupunha, pupunheira e pupunha verde-amarela, pertence à família das Aricaceae. Os primeiros povos europeus, diziam que a sua domesticação começou pelo interesse de fazer utensílios para a caça devido a sua madeira ser rígida e pelos seus produtos, o palmito. A partir de 1970 o interesse pela cultura começou a crescer, tendo em vista as qualidades agronômicas, ecológicas, industriais e comerciais. A pupunheira por ser uma cultura perene, tem uma elevada exigência das propriedades físicas e químicas do solo e quando produzida em boas condições, 
apresenta rápido crescimento e um palmito de boa qualidade. O Brasil é o maior produtor e consumidor de palmito pupunha no mundo, tendo as maiores áreas plantadas na São Paulo, Bahia, Espírito Santo, Rio de Janeiro, Tocantins, Paraná e Santa Catarina (SANTOS \& LUZ 2007).

O Estado de São Paulo possui destaque como um dos maiores produtores de pupunha do país. A maior concentração está nos municípios de Registro, Iguape, Sete Barras, Jacupiranga, Cajati e Juquiá, municípios concentrados no Vale do Ribeira (IBGE 2009).

A cultura da pupunha foi introduzida na região do vale do Ribeira como forma de auxiliar as comunidades locais com a retirada do palmito para melhoria de aquisição de recursos financeiros (BOVI et al. 2007).

Em relação ao plantio da pupunheira deve-se levar em consideração a densidade de plantas. De acordo com MORA-URPI et al. (1997), não é conhecida uma distância de plantio que atenda todas as necessidades da cultura, como, fertilidade do solo, luminosidade, distribuição de chuvas, temperatura, uso de fertilizantes e corretivos, variabilidade genética e mercado. Em plantios adensados, a produção pode cair levando em conta o sombreamento das plantas, a competição entre essas, disponibilidade de água, nutrientes, doenças, pragas, disponibilidade de luz, entre outros fatores (KULCHETSCKI et al. 2001). As informações biométricas de pupunheira são escassas e encontram-se ainda dispersas em publicações antigas, foram analisados alguns parâmetros sobre essa cultura, levando em consideração a necessidade de suprir essa deficiência. Diante disso, é indispensável o estudo da correlação entre características biométricas das hastes e o rendimento da produção da pupunha.

Dessa forma o presente trabalho objetivou avaliar características de desenvolvimento vegetativo das plantas de pupunheira e produtividade de palmito em função do momento de corte das hastes em cultivo com diferentes densidades populacionais, para o segundo ano de corte.

\section{MATERIAL E MÉTODOS}

O trabalho foi instalado na fazenda do Campus Experimental de Registro da UNESP, coordenadas $24^{\circ} 32^{\prime} 08^{\prime \prime} \mathrm{S}$ e $47^{\circ} 51^{\prime} 55^{\prime \prime} \mathrm{N}$, com altitude de $25 \mathrm{~m}$ e clima do tipo Cfa subtropical úmido com verão quente, conforme a classificação de Köppen, com temperatura média de $22{ }^{\circ} \mathrm{C}$ e precipitação anual de $1129 \mathrm{~mm}$.

$O$ delineamento experimental utilizados foi 0 de blocos casualizados em esquema fatorial $2 \times 5$ e quatro repetições, sendo os tratamentos com cinco alturas de corte do estipe $(1,60 \mathrm{~m}, 1,90 \mathrm{~m}, 2,20 \mathrm{~m}, 2,50$ $\mathrm{m}$ e $2,80 \mathrm{~m}$ ) e duas densidades de plantas (5.000 e 10.000 plantas ha $\left.^{-1}\right)$.

O tratamento com as densidades de plantas foi executado no momento do plantio das mudas de pupunheira, em 24 de janeiro de 2012, sendo que espaçamento utilizado para a instalação do experimento foi de um metro entre covas na linha e dois metros entre linhas de plantio. Em cada cova, a depender do tratamento, foram instaladas uma ou duas mudas resultando nas populações pretendidas. Cada parcela experimental contou com uma população de 40 ou 80 plantas, sendo elas dispostas em quatro linhas de plantio e 10 covas por linha (10 ou 20 plantas por linha).

Os dados meteorológicos foram obtidos dos meses de outubro de 2015 a setembro de 2016, onde a maior precipitação $(252 \mathrm{~mm}$ ) foi referente ao mês de janeiro de 2016, no mês de fevereiro de 2016 obteve maior temperatura média. (Figura 1). Dessa forma verificou-se inverno com temperaturas amenas e precipitação em todos os meses.

A caracterização do tipo de solo está definindo como sistema de planícies e terraços fluviais do Ribeira do Iguape, descrito como terreno planos no baixo ribeira e/ou região da depressão tectônica do Ribeira, de sedimentos modernos, em solos aluviais argilosos do tipo Cambissolos Eutróficos em áreas de montante e hidromórficos eutróficos em solos de planície.

Antes do plantio das mudas de pupunha, em novembro de 2011 foi realizada amostragem do solo na profundidade de 0-0,2 $\mathrm{m}$ para caracterização das seguintes propriedades químicas, de acordo com RAIJ et al. (2001) $\mathrm{pH}\left(\mathrm{CaCl}_{2}\right)=4,3$; Presina $=5 \mathrm{mg} \mathrm{dm}^{-3} ; \mathrm{M} . \mathrm{O} .=46 \mathrm{~g} \mathrm{dm}^{-3} ; \mathrm{K}=1,5 \mathrm{mmolc} \mathrm{dm}{ }^{-3} ; \mathrm{Ca}=15 \mathrm{mmolc} \mathrm{dm}^{-3}$; $\mathrm{Mg}=8 \mathrm{mmolc} \mathrm{dm}^{-3} ; \mathrm{H}+\mathrm{Al}=53 \mathrm{mmolc} \mathrm{dm}^{-3} ; \mathrm{Al}=8 \mathrm{mmolc} \mathrm{dm}^{-3} ; \mathrm{CTC}=77 \mathrm{mmolc} \mathrm{dm}^{-3}$ e $\mathrm{V}=32 \%$. A amostragem do solo na profundidade de $0,2-0,4 \mathrm{~m}$ revelou os valores: $\mathrm{pH}\left(\mathrm{CaCl}_{2}\right)=4,0$; Presina $=1 \mathrm{mg} \mathrm{dm}^{-3}$; M.O. $=28 \mathrm{~g} \mathrm{dm}^{-3} ; \mathrm{K}=0,6 \mathrm{mmolc} \mathrm{dm}^{-3} ; \mathrm{Ca}=7 \mathrm{mmolc} \mathrm{dm}{ }^{-3} ; \mathrm{Mg}=3 \mathrm{mmolc} \mathrm{dm}{ }^{-3} ; \mathrm{H}+\mathrm{Al}=57 \mathrm{mmolc} \mathrm{dm}^{-3} ; \mathrm{Al}=13$ mmolc $\mathrm{dm}^{-3}$; CTC $=67 \mathrm{mmolc} \mathrm{dm}^{-3}$ e V $=15 \%$

O preparo do solo foi realizado por meio de subsolagem e gradagem aradora em 09 de janeiro de 2012. Na sequência foi realizada a aplicação de calcário dolomítico com 1,5 t ha ${ }^{-1}$ (BOVI \& CANTARELLA 1997), e em seguida realizada gradagem niveladora.

A adubação de plantio foi realizada com $150 \mathrm{~g}$ do formulado 04-14-08 (NPK) por cova (750 kg ha-1) (BOVI \& CANTARELLA 1997). 


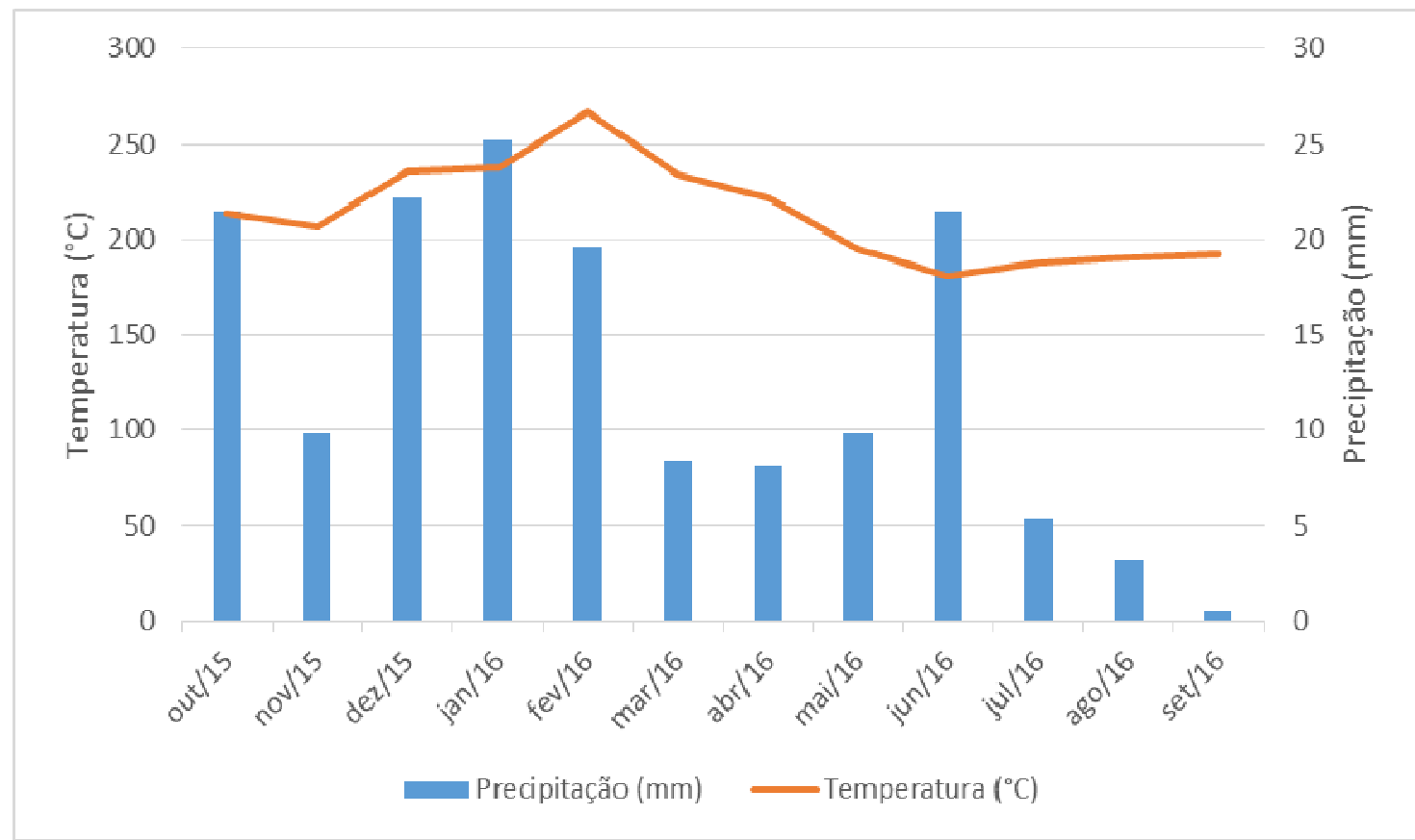

Figura 1. Temperatura mínima, média, máxima e precipitação nos meses de avaliação da pesquisa (março a setembro).

Figure 1. Minimum, average, maximum temperature and precipitation in the months of the research (from March to September).

Para a adubação de formação no primeiro ano de formação (2012) foi aplicado em cobertura, na projeção da copa da planta, $60 \mathrm{~kg} \mathrm{~N} \mathrm{ha}^{-1} ; 30 \mathrm{~kg} \mathrm{P}_{2} \mathrm{O}_{5} \mathrm{ha}^{-1}, 40 \mathrm{~kg} \mathrm{~K}_{2} \mathrm{O} \mathrm{ha}^{-1}$ e $1.250 \mathrm{~kg} \mathrm{FTE} \mathrm{banana} \mathrm{ha}^{-1}$ (Ca $7 \%$, S 5,7\%, B1\%, Cu 1\%, Mn 1\%, Mo 0,1\% e Zn 18\%), sendo as aplicações realizadas em abril, agosto e novembro de 2012 dividida a quantidade total dividida em cada aplicação. No segundo ano de formação das mudas (2013) foi aplicado em cobertura, na projeção da copa da planta, $100 \mathrm{~kg} \mathrm{~N} \mathrm{ha}^{-1} ; 50 \mathrm{~kg} \mathrm{P}_{2} \mathrm{O}_{5} \mathrm{ha}^{-1}, 60$ $\mathrm{kg} \mathrm{K}_{2} \mathrm{O} \mathrm{ha}{ }^{-1}, 1.000 \mathrm{~kg}$ FTE banana ha ${ }^{-1}$ e $1000 \mathrm{~kg}$ calcário dolomítico ha ${ }^{-1}$ (BOVI \& CANTARELLA 1997) aplicando-se em fevereiro, maio, setembro e dezembro de 2013, divididas da mesma forma do ano anterior. No terceiro e quarto ano de manutenção das mudas (2014 e 2015) foi aplicado em cobertura, na projeção da copa da planta, $60 \mathrm{~kg} \mathrm{~N} \mathrm{ha}^{-1} ; 20 \mathrm{~kg} \mathrm{P}_{2} \mathrm{O}_{5} \mathrm{ha}^{-1}, 40 \mathrm{~kg} \mathrm{~K}_{2} \mathrm{O} \mathrm{ha}^{-1}$ (BOVI \& CANTARELLA 1997) aplicando-se em fevereiro, maio, setembro e dezembro, divididas da mesma forma do ano anterior. O primeiro ano com corte das hastes foi em 2015, sendo este realizado em todas hastes oriundas das mudas que foram plantadas.

A fitometria das hastes no segundo ano de corte no período de verão foi realizada nos dias 16 e 17 de março de 2016, e no período de inverno foram realizadas nos dias 01 e 19 de setembro de 2016. Nesse corte as hastes já eram provindas dos primeiros perfilhos emitidos pela muda que foi plantada (segundo corte). Foram avaliadas 12 plantas na área central das parcelas, considerando: altura das hastes, determinada pela distância entre a base da haste até à bifurcação entre folha bandeira e primeira folha aberta (YUYAMA et al. 2002), número de folhas e de perfilhos por planta, por contagem direta e diâmetro do troco da estipe $(\mathrm{cm})$ mensurado a $0,5 \mathrm{~m}$ em relação ao solo.

A determinação de produtividade de palmito também foi realizada nessas datas, por meio do corte de hastes de pupunheira, que estavam no segundo ano de produção e de acordo com as alturas pretendidas. Para o corte das hastes no tratamento com população de plantas de 10.000 ha $^{-1}$ foram cortadas duas hastes por touceira, sendo a produtividade provinda dessas duas plantas.

Após o corte, cada palmito foi fracionado em tolete (parte mais nobre do palmito pupunha) (Figura $2 \mathrm{~A}$ ), rodela (fração que fica entre a tolete e o picado) (Figura $2 \mathrm{~B}$ ) e picado (fração mais basal da haste) (Figura 2C), de acordo com especificações da indústria de processamento de palmito do município de Registro, SP, sendo estas porções pesadas ( $\mathrm{g}$ ) em balança digital e determinado também o rendimento total, somando-se as três porções.

Os dados foram analisados estatisticamente, utilizando análise de variância pelo teste "F". As médias do fator densidade de plantas foram comparadas pelo teste de Tukey a $5 \%$ de probabilidade e para o fator altura de corte, a regressão polinomial sendo adotado a função de maior grau. Foi utilizado o programa estatístico SISVAR 5.6 (FERREIRA 2011). 


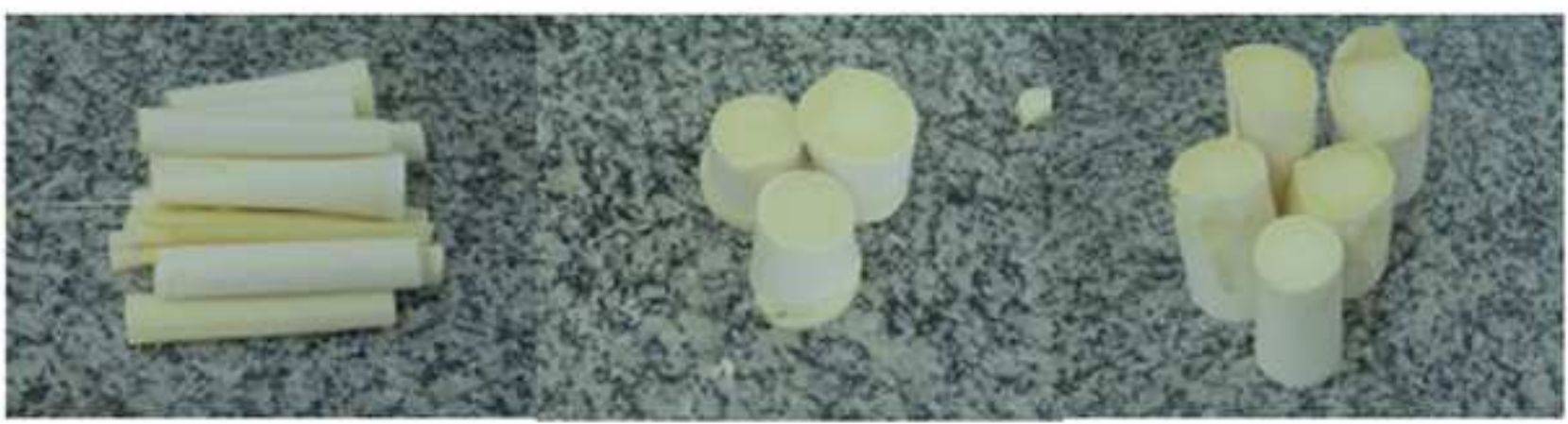

Figura 2. Palmito pupunha proveniente do fracionamento, pupunha fracionado em tolete $(A)$, pupunha fracionado em rodela (B), e pupunha fracionado picado (C).

Figure 2. Pejibaye heart-of-palm from fractionation, pejibaye fractionated on thole $(A)$, pejibaye fractionated on slices $(B)$, and pejibaye fractionated into minces $(C)$.

\section{RESULTADOS E DISCUSSÃO}

Verificou-se que hastes mais altas obteve o número maior de folhas em relação as demais alturas, tendo as médias de forma linear (Tabela 1).

Da mesma forma o diâmetro das hastes aumentou de forma linear em função da altura das hastes.

Ao avaliar a população de plantas verificou-se maior diâmetro das hastes com a menor população avaliada. MORAES NETO et al. (2000) afirmam que várias características constituem parâmetros para avaliar as respostas de crescimento de hastes à intensidade luminosa. Dentre essas, a de uso mais frequente é a altura da haste, visto que a capacidade em crescer rapidamente quando sombreadas é um mecanismo de aclimatação das plantas, compreendendo uma valiosa estratégia para escapar do sombreamento. Nesse sentido, KULCHETSCKI et al. (2001) garantem que plantios mais adensados produzem palmitos mais finos, indicando, assim, a necessidade de manejo das plantas, caso o mercado exija palmito com maior diâmetro.

A altura de corte da haste em 2,32 m proporcionou o máximo rendimento em palmito picado (peso). E em relação ao rodela e tolete não houve influência dos tratamentos. Contudo, a produção de palmito (picado + rodela + tolete) aumentou de forma linear com a altura de plantas. De acordo com a equação ajustada este aumento foi de $155 \mathrm{~g}$ de palmito a cada metro de altura da haste. Dessa forma hastes mais altas produzem maior quantidade de palmito. A população de plantas não influenciou as características produtivas.

O resultado referente as hastes conduzidas com a população de plantas de (5.000 plantas por hectare), obteve maior quantidade de folha em relação a outra população de plantas. O número de perfilhos por planta, não foi influenciado pelos tratamentos. (Tabela 2).

O diâmetro das hastes aumentou de forma linear em função da altura das hastes. É importante salientar uma dinâmica de crescimento diferente das hastes de pupunha, pois as que foram plantadas (primeiro corte) crescem mais em altura e menor em diâmetro do colmo, enquanto que as hastes originadas a partir de perfilhos (segundo corte) crescem menos em altura e mais em diâmetro, num mesmo período de tempo.

Hastes conduzidas na altura de corte de 2,8 m obtiveram maiores quantidades de palmito processado em picado, rodela, tolete e total. Sendo picado $1006,25 \mathrm{~g}$ touceira $^{-1}$, rodela $278,75 \mathrm{~g}$ touceira $^{-1}$, tolete $1.042,50 \mathrm{~g}$ touceira $^{-1}$ e total $2.327,50 \mathrm{~g}_{\text {touceira }}{ }^{-1}$. $\mathrm{E}$ em relação à densidade populacional houve diferença significativa para o palmito processado em rodela.

Segundo MORA-URPI et al. (1997), não existe uma distância ótima de plantio permanente que atenda todas circunstâncias que envolvem o cultivo de pupunheira, tais como: condições de fertilidade do solo, distribuição de chuvas, luminosidade, temperatura, uso de fertilizantes, variabilidade genética e mercado. Nos plantios adensados, a produção pode cair ao longo do tempo, principalmente em razão do sombreamento e, também por causa da competição entre plantas que, além de limitar o desenvolvimento de perfilhos, aumentar a demanda por luz, água e nutrientes. 
Tabela 1. Valores de $\mathrm{p}>\mathrm{F}$ e teste de comparação de médias para desenvolvimento vegetativo e rendimento de palmito de pupunheira de acordo com população de plantas e alturas de corte. Registro, SP, segundo ano de produção, março de 2016.

Table 1. Values of $p>F$ and comparison test of the averages for crop development and yield of the pejibaye heart-of-palm according to the plants population and cutting height. Registro, SP, second year of production, March of 2016.

\begin{tabular}{|c|c|c|c|c|c|c|c|}
\hline Teste $\mathrm{F}$ & Folhas & Diâmetro & Perfilho & $\begin{array}{l}\text { Picado } \\
p>F\end{array}$ & Rodela & Tolete & Total \\
\hline População (p) & 0,994 & 0,000 & 0,052 & 0,261 & 0,271 & 0,227 & 0,232 \\
\hline Altura (a) & 0,004 & 0,000 & 0,832 & 0,007 & 0,787 & 0,842 & 0,021 \\
\hline$p^{*} a$ & 0,707 & 0,274 & 0,920 & 0,275 & 0,828 & 0,534 & 0,537 \\
\hline & (número) & $(\mathrm{mm})$ & (número) & \multicolumn{4}{|c|}{$\left(\mathrm{g}\right.$ touceira $\left.{ }^{-1}\right)$} \\
\hline 5.000 & $5,59 a$ & $110,43 a$ & $4,94 a$ & $432,35 a$ & $73,05 \mathrm{a}$ & $286,95 a$ & $792,35 a$ \\
\hline 10.000 & $5,59 a$ & $91,61 \mathrm{~b}$ & $5,69 a$ & $394,35 a$ & $82,90 \mathrm{a}$ & $242,20 \mathrm{a}$ & $719,45 a$ \\
\hline C.V. \% & 12,50 & 13,77 & 21,92 & 25,33 & 35,59 & 43,31 & 24,95 \\
\hline D.M.S. & 0,454 & 9,024 & 0,757 & 67,925 & 18,007 & 74,349 & 122,353 \\
\hline \multicolumn{8}{|c|}{ Regressão Polinomial } \\
\hline 1,60 & 5,12 & 82,01 & 5,12 & 303,50 & 73,25 & 275,00 & 627,62 \\
\hline 1,90 & 5,58 & 97 & 5,37 & 464,75 & 81,37 & 256,37 & 768,00 \\
\hline 2,20 & 5,12 & 96,36 & 5,70 & 467,00 & 85,50 & 240,12 & 754,12 \\
\hline 2,50 & 5,70 & 104,88 & 5,08 & 504,00 & 69,62 & 250,50 & 770,50 \\
\hline 2,80 & 6,45 & 123,89 & 5,32 & 422,50 & 80,12 & 300,87 & 859,25 \\
\hline $\mathrm{p}>\mathrm{F}$ (linear) & $0,0013^{(1)}$ & $0,0000^{(2)}$ & 0,9259 & 0,1055 & 0,9491 & 0,7231 & $0,0358^{(4)}$ \\
\hline p>F (quadrática) & 0,0908 & 0,3859 & 0,5371 & $0,0293^{(3)}$ & 0,6812 & 0,2871 & 0,7720 \\
\hline r2 (linear \%) & 65,05 & 88,36 & 0,61 & 31,92 & 0,24 & 9,19 & 78,89 \\
\hline r2(quadrática \%) & 80,72 & 90,38 & 27,46 & 92,18 & 10,32 & 93,74 & 80,27 \\
\hline
\end{tabular}

Equações Polinomiais

(1) $Y=3,5466+0,9320 x{ }^{(2)} Y=34,5547+30,2150 x^{(3)} Y=-1438,0261+1666,4642 x+357,7380 x^{2}$

${ }^{(4)} \mathrm{Y}=414,3500+155,2500 \mathrm{x}$

*Médias seguidas pela mesma letra não diferem entre si pelo teste de Tukey a $5 \%$ de probabilidade.

Tabela 2. Valores de $\mathrm{p}>\mathrm{F}$ e teste de comparação de médias para desenvolvimento vegetativo e rendimento de palmito de pupunheira de acordo com população de plantas e alturas de corte. Registro, SP, segundo ano de produção, setembro de 2016.

Table 2. Values of $p>F$ and comparison test of the averages for crop development and yield of the pejibaye heart-of-palm according to the plants population and cutting height. Registro, SP, second year of production, September of 2016.

\begin{tabular}{|c|c|c|c|c|c|c|c|}
\hline Teste F & Folhas & Perfilho & Diâmetro & $\begin{array}{l}\text { Picado } \\
p>F\end{array}$ & Rodela & Tolete & Total \\
\hline População (p) & 0,002 & 0,923 & 0,304 & 0,579 & 0,000 & 0,078 & 0,0794 \\
\hline Altura (a) & 0,000 & 0,170 & 0,000 & 0,115 & 0,000 & 0,000 & 0,0016 \\
\hline$p^{*} a$ & 0,605 & 0,856 & 0,301 & 0,944 & 0,045 & 0,555 & 0,8949 \\
\hline & (número) & (número) & $(\mathrm{mm})$ & \multicolumn{4}{|c|}{$\left(\mathrm{g}\right.$ touceira $\left.{ }^{-1}\right)$} \\
\hline 5.000 & $5,84 a$ & $4,36 \mathrm{a}$ & $98,80 a$ & $864,25 a$ & $255,00 a$ & $835,50 \mathrm{a}$ & $1954,75 \mathrm{a}$ \\
\hline 10.000 & $5,24 b$ & $4,39 \mathrm{a}$ & $101,73 \mathrm{a}$ & $818,25 a$ & $179,00 \mathrm{~b}$ & $745,00 \mathrm{a}$ & $1742,25 \mathrm{a}$ \\
\hline C.V. \% & 10,10 & 23,49 & 8,83 & 30,86 & 19,02 & 19,83 & 19,94 \\
\hline D.M.S. & 0,363 & 0,667 & 5,742 & 168,431 & 26,783 & 101,665 & 239,1864 \\
\hline & \multicolumn{7}{|c|}{ Regressão Polinomial } \\
\hline 1,60 & 4,91 & 5,03 & 87,15 & 748,75 & 185,62 & 667,50 & 1601,87 \\
\hline 1,90 & 5,03 & 4,53 & 91,64 & 718,12 & 177,50 & 647,50 & 1543,12 \\
\hline 2,20 & 5,24 & 3,83 & 99,08 & 775,00 & 226,87 & 809,37 & 1811,25 \\
\hline 2,50 & 6,08 & 4,49 & 108,48 & 958,12 & 216,25 & 784,37 & 1958,75 \\
\hline 2,80 & 6,45 & 3,99 & 114,98 & 1006,25 & 278,75 & 1042,50 & 2327,50 \\
\hline p>F (linear) & $0,0000^{(1)}$ & 0,0763 & $0,0000^{(2)}$ & $0,0149^{(3)}$ & $0,0000^{(4)}$ & $0,0000^{(5)}$ & $0,0001^{(6)}$ \\
\hline p>F (quadrática) & 0,1377 & 0,3186 & 0,6138 & 0,4159 & 0,1484 & 0,0860 & 0,1437 \\
\hline r2 (linear \%) & $91,44 \%$ & $48,86 \%$ & $98,83 \%$ & $82,62 \%$ & $78,42 \%$ & $79,07 \%$ & $87,78 \%$ \\
\hline r2 (quadrática \% ) & $96,37 \%$ & $63,70 \%$ & $99,31 \%$ & $90,96 \%$ & $85,72 \%$ & $88,87 \%$ & $97,48 \%$ \\
\hline
\end{tabular}

Equações Polinomiais

${ }^{(1)} Y=2,5190+1,3762 x{ }^{(2)} Y=47,1083+24,1641 x{ }^{(3)} Y=287,5833+251,6666 x{ }^{(4)} Y=52.0000+75,0000 x$ ${ }^{(5)} Y=139,8750+295,6250 x{ }^{(6)} Y=479,4583+622,2916 x$ 
Foi possível observar que, na avaliação de setembro, o rendimento total do palmito pupunha é maior em relação a avaliação de março. Isso ocorre devido ao corte das hastes em março, desenvolveram-se no período da primavera e verão, com temperaturas altas. Para corte em setembro, as hastes desenvolveramse nas estações de outono e inverno, com temperaturas amenas e precipitação adequada, condições estas que devem ter favorecido o desenvolvimento das plantas resultando em maior produtividade de palmito.

A pupunha tem mostrado adaptação a diversas condições edafoclimáticas. Diversos autores indicam, que a precipitação deve ser superior a 1900 - 2000 mm/ano (BLAAK 1976, FLORES \& GOMES 1986, CLEMENT 1989, SILVA et al. 1991, MORA-URPI 1992, WOLF 1998), mas há algumas referências observando que a quantidade anual de chuva deve ser maior que 1500 - $1600 \mathrm{~mm}$ (VILLACHICA 1996, BONACCINI 1997, BOVI 1998, CLEMENT 1998). Além da precipitação pluviométrica total, é importante que as chuvas sejam bem distribuídas no ano; sendo que períodos de três a quatro meses de seca podem afetar o desenvolvimento e produtividade da pupunha (BLAAK 1976, MORA-URPI 1984, CLEMENT 1989, VILLACHICA 1996, BOVI 1998). A temperatura média anual indicada como mais adequada para desenvolvimento da palmeira, varia de 18 a $28{ }^{\circ} \mathrm{C}$. Entretanto, temperaturas médias anuais entre 24 a $28^{\circ} \mathrm{C}$ parecem ser mais favoráveis à espécie. Além disso, diversos autores citam que $B$. gasipaes é pouco ou nada tolerante a geadas (JOHANNESSEN 1966, FLORES \& GOMES 1986, VILLACHICA 1996, BONACCINI 1997, BOVI 1998).

\section{CONCLUSÃO}

Hastes de pupunheira colhidas no mês de março e com altura de 2,8 m obtém maior diâmetro do caule, número de folhas e quantidade total de palmito produzido. Corte realizado em setembro com hastes de $2,8 \mathrm{~m}$ de altura proporciona maior produção de palmito picado, rodela, tolete e quantidade total de palmito pupunha.

\section{AGRADECIMENTOS}

Viveiro MM Mudas e ao Engenheiro Agrônomo Marcio Franchetti, que doou as mudas de pupunha para instalação do projeto de pesquisa.

\section{REFERÊNCIAS}

BLAAK G. 1976. Pejibaye. Abstracts on Tropical Agriculture 2: 9-17.

BONACCINI LA. 1997. Produza palmito: A cultura da pupunha. Cuiabá: SEBRAE. 100p.

BOVI MLA. 2007. Lodo de esgoto e produção de palmito em pupunheira. Revista Brasileira de Ciência do Solo 31: 153166.

BOVI MLA. 1998. Palmito pupunha: informações básicas para cultivo. Campinas: Instituto Agronômico. 50p. (Boletim 100).

BOVI MLA \& CANTARELLA H. 1997. Pupunha para extração de palmito. In: RAIJ BV et al. Recomendações de adubação e calagem para o Estado de São Paulo. 2.ed. Campinas: Instituto agronômico e Fundação IAC. 285p.

CLEMENT CR. 1998. Introdução pupunha. Disponível em: http://www. inpa.gov.br/artigos/crd.html. Acesso em: 20 jan. 2018.

CLEMENT CR. 1989. The potencial use of the pejibaye palm in agroforestry systems. Agroforestry Systems 7: 201-212.

FERREIRA DF 2011. SISVAR: A computer statistical analysis system. Ciência e Agrotecnologia 35: 1039-1042.

FLORES WBC \& GOMES JBM. 1986. A pupunha (Bactris gasipaes H.B.K.): cultura e manejo. Manaus: INPA.

IBGE. 2009. Instituto Brasileiro de Geografia e Estatística. Censo agropecuário - Resultados preliminares. Rio Janeiro: IBGE.

JOHANNESSEN CL. 1966. Pejibayes in commercial production. Turrialba 16: 181-187.

KULCHETSCKI L et. al. 2001. Palmito Pupunha (Bactris gasipaes Kunth): a espécie, cultura, manejo agronômico, usos e processamentos. Ponta Grossa: UEPG. 148p.

MORAES NETO SP et al. 2000. Crescimento de mudas de algumas espécies arbóreas que ocorrem na mata atlântica em função do nível de luminosidade. Revista Árvore 24: 35-45.

MORA-URPI J. 1984. Pejibaye (Bactris gasipaes H.B.K.): origem, biologia floral y manejo agronômico. In: MORA-URPI J. (Ed.) Palmeras poço utilizadas de América tropical. Turrialba: FAO/CATIE. p.118-160.

MORA-URPI et al. 1997. Peach palm (Bactris gasipaes Kunth). Gatersleben: International Plant Genetics Resourch Institute. 20p.

MORA-URPI J. 1992. Pejibaye. (Bactris gasipaes). In: HERNANDEZ B \& LEON J. (Eds.). Cultivos marginados. Otra perspectiva de 1492 Roma: FAO. p.209-219.

SANTOS AF dos \& LUZ EDMN. 2007. Doenças emergentes causadas por Phytophthora. Fitopatologia Brasileira 32: 4142.

SILVA AF et al. 1991. Seminário: a pupunheira e suas potencialidades econômicas. Manaus: Secretaria de Estado da Produção Rural e Abastecimento. 
VILLACHICA H. 1996. Pijuayo. Bactris gasipaes H.B.K. In: VILLACHICA H. (Ed). Frutales y hortalizas promisorios de la Amazônia. Lima: Tratado de Cooperación Amazônica. p.216-226.

WOLF M. 1998. Pupunha. Disponível em: http://home.braunschweig.netsurf.de/andree.wof/pupunha.html. Acesso em: 05 jan. 2018.

YUYAMA K et al. 2002. Pupunheira. In: BRUCKNER CH. (Ed.) Melhoramento de fruteiras tropicais. Viçosa: UFV. p.411422. 